\section{CD24 expression predicts disease progression in prostate cancer}

As part of the search for new prognostic markers in prostate cancer, Kristiansen et al. have studied the expression of CD24, a small, glycosylphosphatidylinositol-linked cell surface protein thought to be involved in metastasis.

Using tissue from 102 adenocarcinomas of the prostate and 31 nodal metastases, the investigators assessed the level of expression of CD24 immunohistochemically, and assigned a semiquantitative score for each sample. They then related these findings to clinicopathologic parameters measured during the median follow-up period of 30.5 months.

Although rare in normal tissue, CD24 expression was observed in almost half $(48 \%)$ of the primary prostate cancer samples. Furthermore, the protein was detected in $68 \%$ of the nodal metastases. In a multivariate analysis, CD24 expression in primary tumors strongly predicted earlier disease progression-as indicated by prostate-specific antigen relapse-with a relative risk of $3.2(P=0.005)$. This new prognostic marker appeared to be a more influential predictor of disease progression than $\mathrm{pT}$ stage or preoperative prostate-specific antigen level.

Concluding that the measurement of CD24 expression might provide a useful means of risk stratification in prostate cancer, the authors remark that the protein is overexpressed in several other solid tumors and so may have a role as a general prognostic tumor marker.

Original article Kristiansen G et al. (2004) CD24 expression is a significant predictor of PSA relapse and poor prognosis in low grade or organ confined prostate cancer. Prostate 58: 183-192

\section{Systemic inflammatory response is a prognostic factor in bladder cancer}

Evidence is mounting about the role of the systemic inflammatory response in cancer progression. A recent study published in the British Journal of Cancer explores its relationship with survival in 105 patients with transitional cell carcinoma of the urinary bladder.

C-reactive protein levels were determined in all patients before and/or after transurethral resection of the bladder tumor; elevated circulating concentrations of C-reactive protein (>10 mg/l) indicated a systemic inflammatory response. During the follow-up period, both preoperative and postoperative elevated C-reactive protein concentrations were significantly associated with overall survival, as were tumor stage and grade. A multivariate analysis of the 59 patients who had C-reactive protein levels measured preoperatively showed that elevated C-reactive protein was independently associated with cancer-specific survival (hazard ratio 3.31, 95\% Cl 1.09-10.09).

In summary, this study showed that the systemic inflammatory response, as indicated by elevated levels of C-reactive protein, predicted cancer-specific survival independently of tumor stage or grade. The authors discuss possible mechanisms by which this effect might be mediated and call for further studies in this area.

Original article Hilmy M et al. (2005) The relationship between the systemic inflammatory response and survival in patients with transitional cell carcinoma of the urinary bladder. Br J Cancer 92: 625-627

\section{Menopausal hormone therapy increases urinary incontinence risk}

Besides the symptomatic relief of hot flashes and other problems associated with the menopause, menopausal hormone therapy is frequently used to treat urinary incontinence. Recent results from two Women's Health Initiative trials, however, have shown that the risk of developing urinary incontinence is actually increased in women receiving this therapy, whether with estrogen alone or estrogen plus progestin. Furthermore, symptoms were worsened after one year of hormone therapy in those who were symptomatic at baseline.

Hendrix et al. studied the effects of menopausal hormone therapy, compared with placebo, in 27,347 healthy, postmenopausal women enrolled between 1993 and 1998 . There were two separate trials, assessing conjugated equine estrogen plus progestin $(n=16,608)$ and estrogen alone $(n=10,739)$. The trials were designed to assess whether these therapies were useful in the prevention of hip fractures and coronary heart disease, 